\title{
Guidewire Fracture, Entrapment in Proximal Left Anterior Descending and Successful Extraction with Snare Catheter
}

\begin{abstract}
A64 year old female patient with a history of dyslipidaemia presented with Canadian Cardiovascular Society class II angina and stage 1 positive treadmill stress test. Coronary angiogram revealed $20 \%$ stenosis in distal left main coronary artery (LMCA), two $95 \%$ stenosis in proximal left anterior descending artery (LAD) and non-significant disease in left circumflex and right coronary arteries.

LAD lesion was crossed with Rinato guidewire and first diagonal branch (D1) crossed with BMW guidewire. Both proximal and distal LAD lesions were predilated and stented with $2.75 \times 38 \mathrm{~mm}$ Xience prime (Everolimus Eluting) stent. After the stent implantation, jailed guidewire used to protect the diagonal branch could not be removed. Traction, pull and push, balloon reinflation and deflation, back-and-fourth vibration were attempted to no avail. Finally guidewire fracture occurred and eventually entrapment leaving a part in D1, LAD and LMCA.

By using an endovascular snare system we were able to remove the fractured guidewire successfully. This case highlights that endovascular snare system can safely and effectively be used for extraction of fractured retained guidewires inside the coronary arteries which prevents impending surgical intervention.
\end{abstract}

Keywords: Guidewire fracture - Entrapment - Snare catheter - PCI

\section{Introduction}

Guidewire fracture and entrapment is a rare but serious complication during percutaneous coronary intervention (PCI). The increasing use of PCI in recent years has made it a notable complication for operators to be aware of. It can lead to thrombus formation, embolism and perforation. There are several ways to manage this complication. These include conservative follow-up, percutaneous removal, stent implantation and surgical removal being the last resort. We present a successful case where snare catheter was used to retrieve the jailed fractured guidewire in a 64 year old female patient who underwent percutaneous transluminal coronary angioplasty (PTCA) to LAD [1-15].

\section{Case Report}

A 64-year-old female with a history of dyslipidemia presented with Canadian Cardiovascular Society class II angina. Her 2D echo was normal with an EF of $>60 \%$. Her treadmill stress test was strongly positive at Stage 1. Coronary angiogram revealed $20 \%$ stenosis in distal left main coronary artery and 95\% proximal stenosis in LAD, followed by $95 \%$ stenosis in at the level of first diagonal branch (Figure1, 2) (Video 1). Left circumflex and right coronary arteries had non-significant disease (Videos 2 and 3).

LAD lesions was crossed with 0.014 Rinnato guidewire and LADD1 crossed with 0.014 BMW (hi-torque Balance Middleweight) guidewire. Both proximal and diatal LAD lesions were predilated with $2.5 \times 15 \mathrm{~mm}$ balloon at $8 \mathrm{~atm}$ and stented with $2.75 \times 38$ $\mathrm{mm}$ Xience prime(Everolimus eluting) stent at 9 atm (Video 4).
Uditha Indika Hewarathna* Ajith Indika Kularatne, Shanike Prasad Karunaratne, Dolapihilla SNB, Thilini

Dayaratne

Department of Cardiology, Teaching Hospital Kandy, Sri Lanka

*Author for correspondence: Email: udithaih@gmail.com; kandycardiology@gmail.com Received date: March 11, 2019 Accepted date: June 14, 2019 Published date: June 21, 2019 


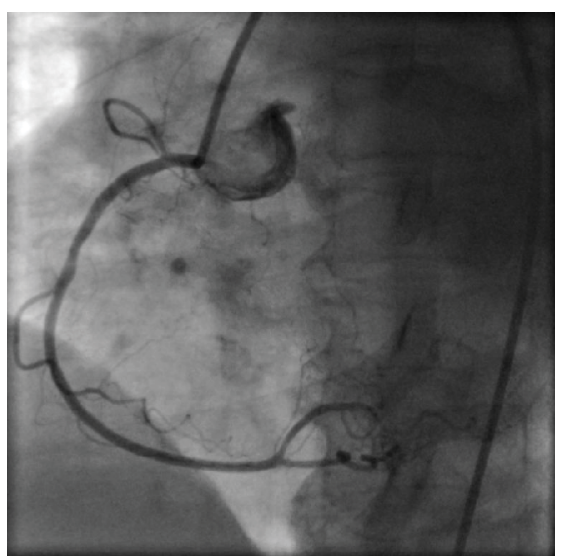

Figure 1: Coronary angiogram showing Right Coronary Artery having the non-significant disease.

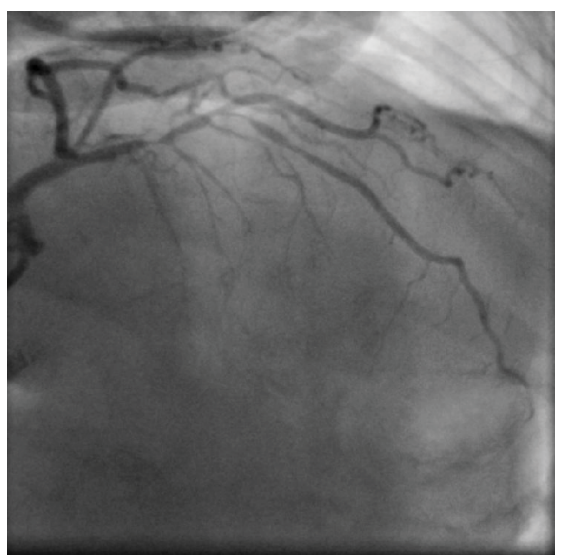

Figure 2: Coronary angiogram showing $20 \%$ stenosis in distal Left Main Coronary Artery, 95\% proximal stenosis in LAD and $95 \%$ stenosis at the level of first diagonal branch.

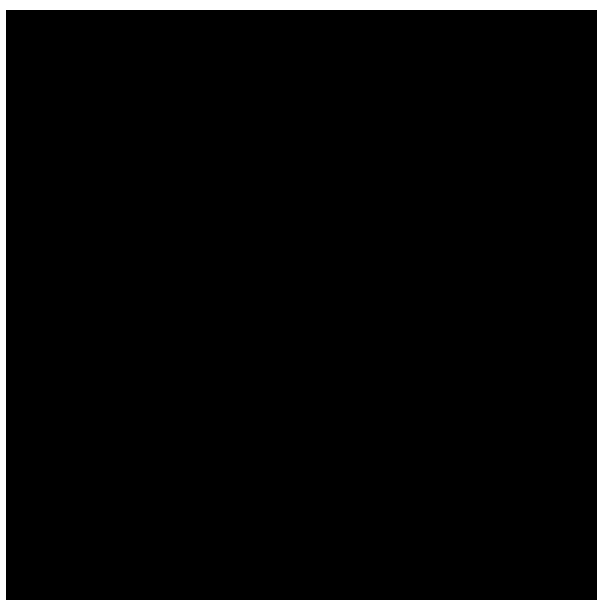

Video 1: Coronary angiogram showing $20 \%$ stenosis in distal Left Main Coronary Artery, 95\% proximal stenosis in LAD and $95 \%$ stenosis at the level of first diagonal branch.

After the stent implantation, guidewire used to protect the diagonal branch could not be removed (Video 5). Subsequently,we made several attempts using simple

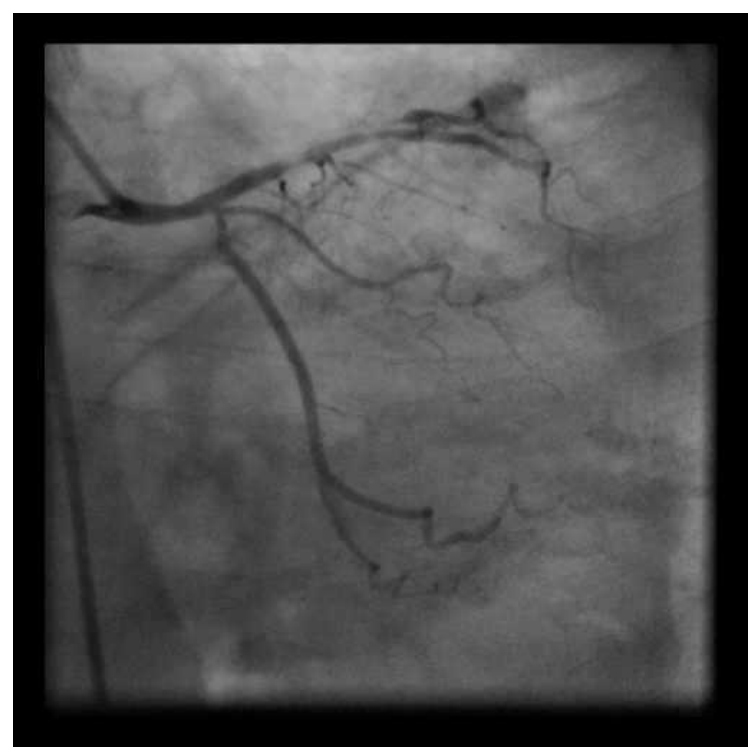

Video 2: Coronary angiogram showing 20\% stenosis in distal Left Main Coronary Artery, 95\% proximal stenosis in LAD and 95\% stenosis at the level of first diagonal branch and Coronary angiogram showing Left Circumflex and Right Coronary Artery having non-significant disease.

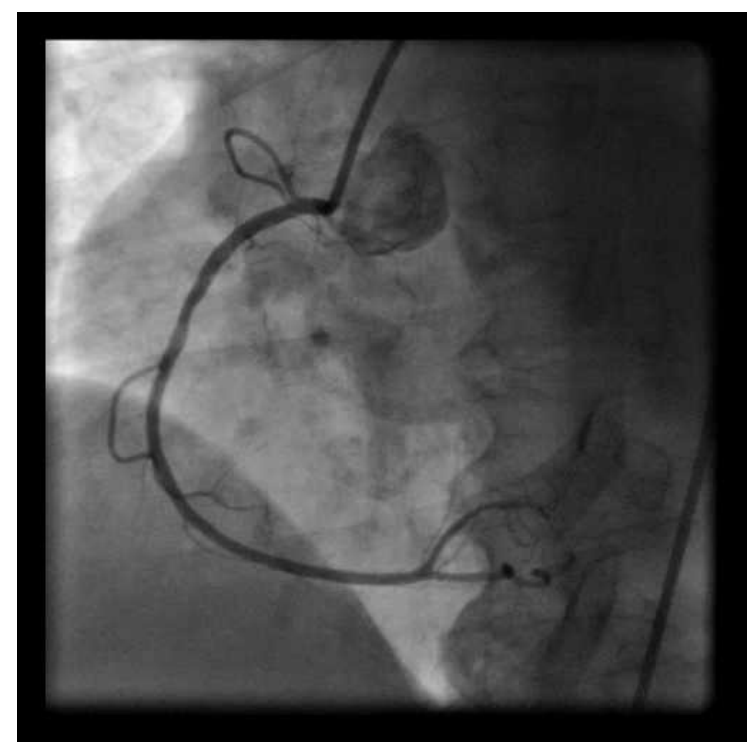

Video 3: Coronary angiogram showing Left Circumflex and Right Coronary Artery having non-significant disease.

manoeuvres like traction, pull and push, balloon reinflation and deflation,back-and-forth vibration without successs. And guide wire was fractured and entrapped leaving part in D1, LAD and LMCA (Figure 3) (Video 6).

We used a GooseNeck loop-snare (Microvena Corporation, St. Paul, MN, USA) to remove the fractured guidewires. With multiple rotatory 


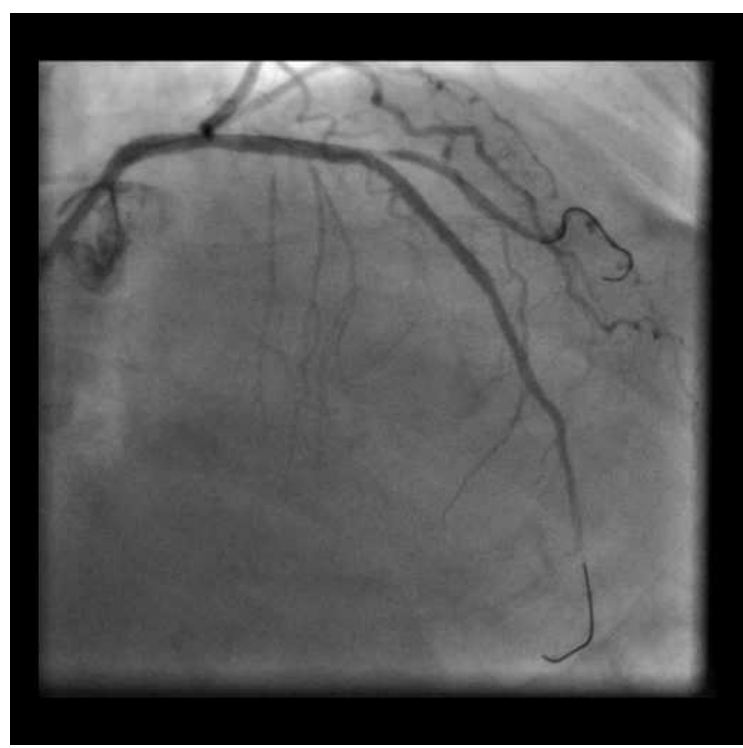

Video 4: Coronary angiogram showing both proximal and distal LAD lesions were predilated with $2.5 \times 15 \mathrm{~mm}$ balloon at $8 \mathrm{~atm}$ and stented with $2.75 \times 38 \mathrm{~mm}$ Xience prime(Everolimus Eluting) stent (Abbott Vascular International) at $9 \mathrm{~atm}$.

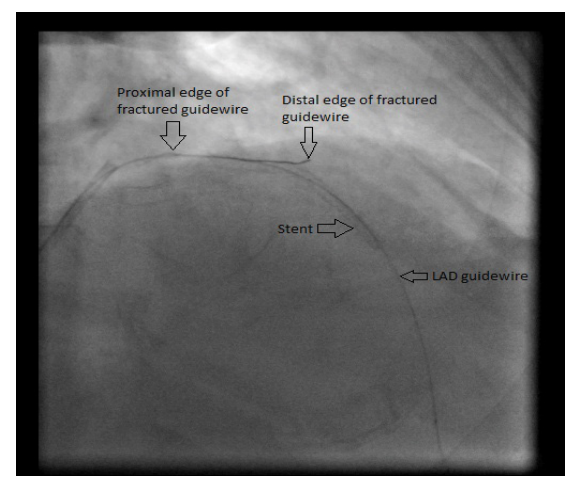

Figure 3: Coronary angiogram showing the guidewire getting fractured and entrapped leaving a part in D1,LAD and LMCA.

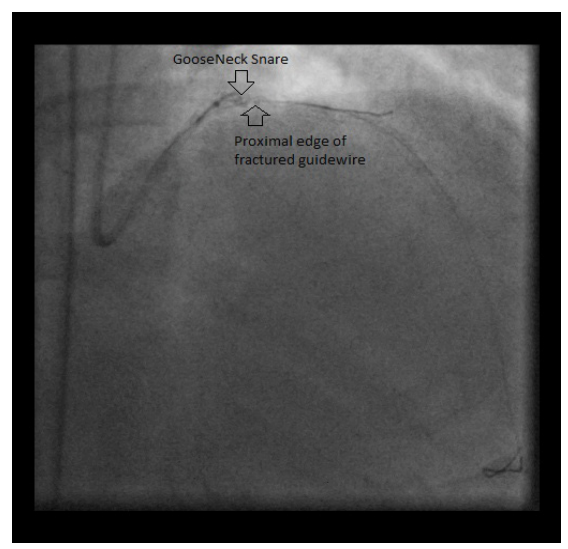

Figure 4: Endovascular Goose Neck Snare to remove remnant.

movements of the snare, we were able to successfully removed the fractured guidewire (Figure 4) (Video 7).

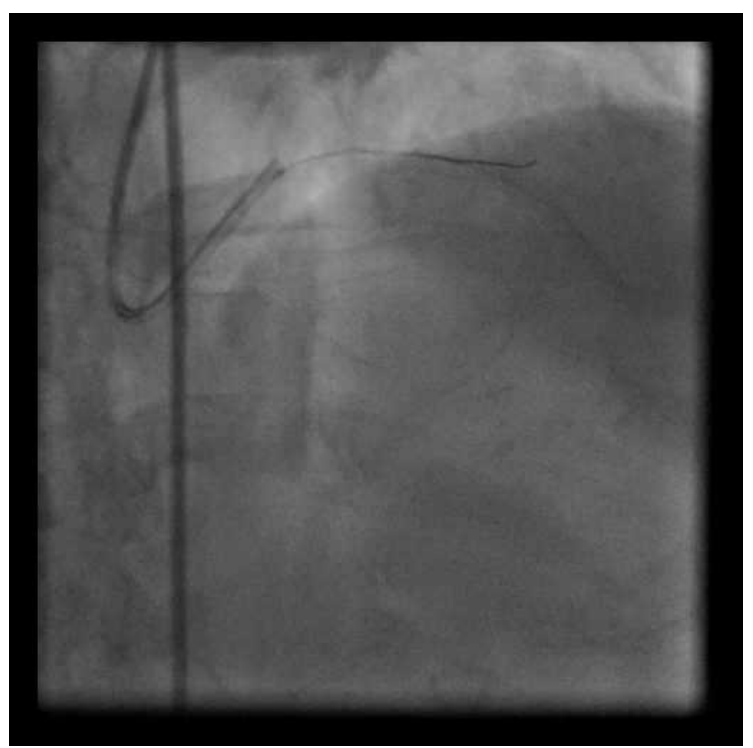

Video 5: Coronary angiogram showing the guidewire which was used to protect the diagonal branch failing to be removed

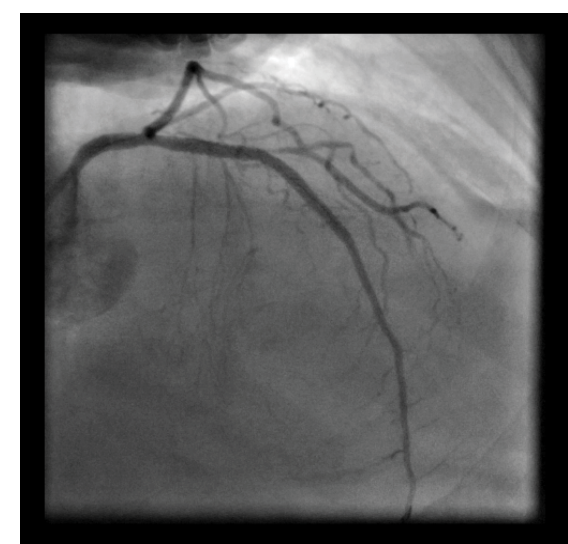

Figure 5: Post-dilatation after removal of guidewire.

After removing the jailed guidewire the stent was post dilated with $3.0 \times 8 \mathrm{~mm}$ balloon at 18 atm proximally and 12 atm distally (Figure 5). Excellent final angiographic results and TIMI III flow were achieved in LAD (Video 8).

\section{Discussion}

Fracture and entrapment of guidewire occur infrequently in percutaneous coronary interventions with only $0.2-0.8 \%$ cases reported during PCI [6]. Coronary artery thrombosis, as well as, embolization are common complications 3-5 of remaining guidewire segments where metallic remnants are more prone to these events compared to hydrophilic non-metallic guidewires [7].

Some of the risk factors for entrapment of guidewire 


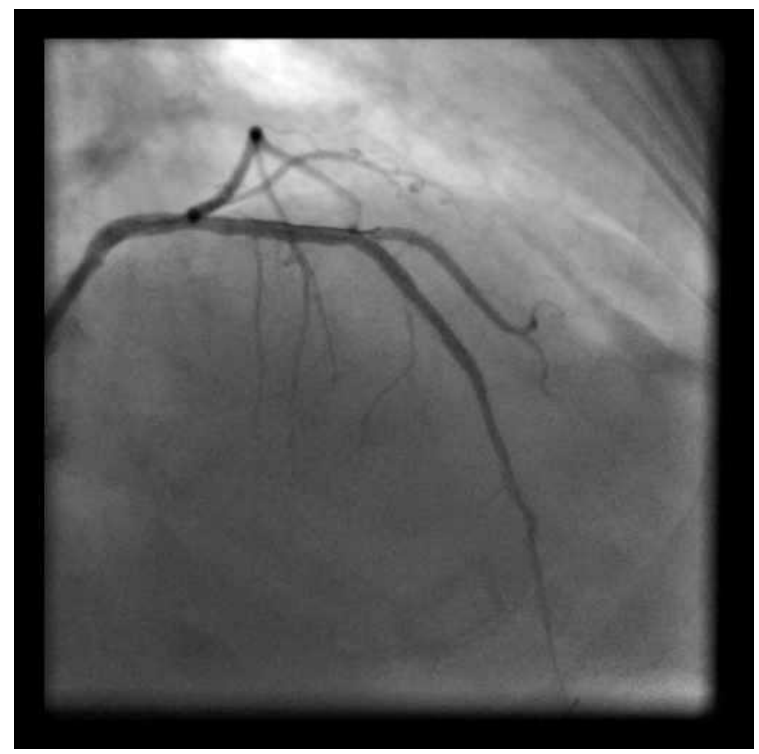

Video 6: Coronary angiogram showing the guidewire getting fractured and entrapped leaving a part in D1,LAD and LMCA

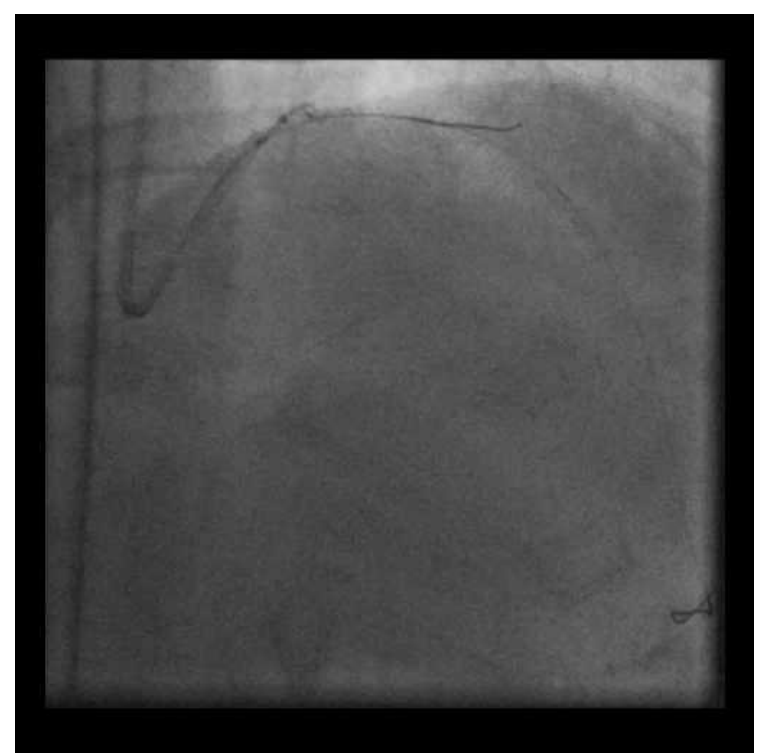

Video 7: Coronary angiogram showing use of a $2-4 \mathrm{~mm} \times 175 \mathrm{~cm}$ Endovasular snare system (Merst Medical Systems,Inc. South Jordan) to remove the fractured guidewire

include firm tipped guidewire usage, sharply curved guidewire tip, repeated excessive maneuvers using balloon or stent catheter, rotablator use, multiple guidewire usage, calcified and tortuous vessels, chronic total occlusion, bifurcation lesion stenting, stent restenosis treatment and prolonged PCI [7]. Treatment options can divide be into conservative management, interventional techniques and surgery.

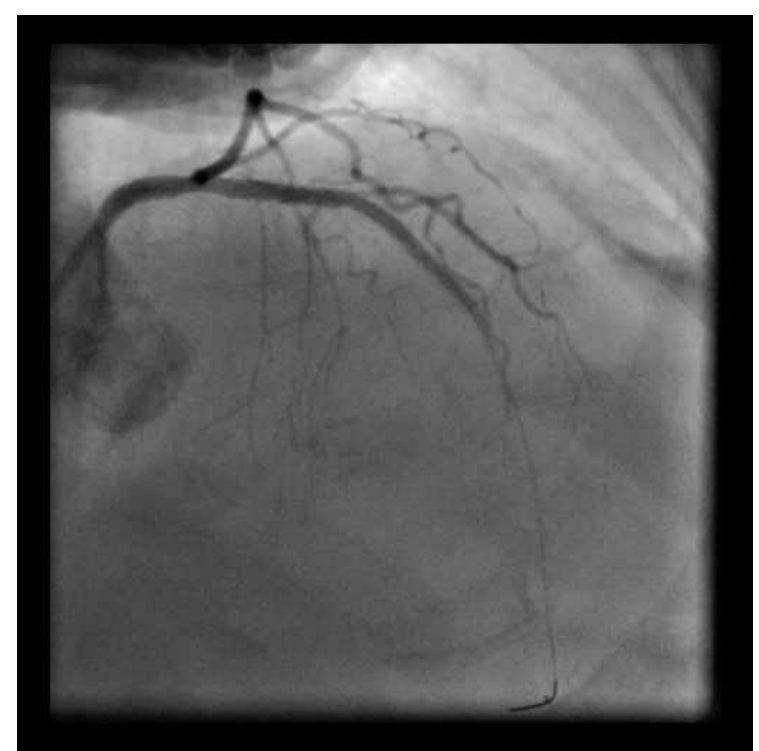

Video 8: Coronary angiogram showing excellent final angiographic results with TIMI III flow achieved in LAD.

Conservative treatment is followed if percutaneous techniques fail, if the patient is haemodynamically stable or if the patient refuses surgery [1,5]. Kaplan et al. reported a case in 2010 where after unsuccessful attempts of retrieval it was decided that the wire should remain in the vessel and the patient received lifelong medical treatment with dual antiplatelets. The patient was symptom free for up to two years [2].

Pourmoghaddas et al. [8] suggest that it is best to leave a remnant in patient provided that it is in a small side branch and the wire is not metallic. However there is a case reported by $\mathrm{Kim}$ et al. in which conservative management lead to subacute stent thrombosis in a 72 year old patient. The patient developed acute chest pain four days later and by day 15 he did not survive [5].

Interventional techniques include extraction with snare catheter, stenting over guidewire, balloon angioplasty over guidewire, mobilization and fixing into small side branch. Snare catheter is the most common and first retrieval technique used by many authors [9-11] where some used GooseNeck snare catheters $[1,5,6]$. GooseNeck snare catheters were also used in guidewire tip retrieval by Kume et al. [12] and in another case it was found that a direct, larger pulling force was possible with a goose snare and it also provided a protection during the pulling process [13].

To avoid further endothelial damage from repeated 
unsuccessful interventional techniques it is recommended that surgery is arranged for such patients. Surgery may involve remnant removal, endarterectomy and bypass grafting $[14,15]$. However, there are no specific guidelines or indications published yet for surgery. In a desperate situation, small snare catheter can be used safely inside the coronary artery to retrieve the guidewire.

\section{Conclusion}

Firmer and more flexible wires are being used in recent years yet guidewire fracture and entrapment is

\section{References}

1. Hong YM, Lee SR. A case of guide wire fracture with remnant filaments in the left anterior descending coronary artery and aorta. Korean Circ J. 40(9): 475-7 (2010).

2. Kaplan S, Kaplan ST, Kutlu M. An unusual case of guide wire fractured during primary percutaneous coronary intervention, and two year follow-up. Kardiologia polska. 68(11): 1291-3 (2010).

3. Demircan S, Yazici M, Durna K, Yasar E. Intracoronary guidewire emboli: a unique complication and retrieval of the wire. Cardiovasc Revas Med. 9(4): 278-80 (2008).

4. Lopez-Minguez JR, Davila E, Doblado M, et al. Rupture and intracoronary entrapment of an angioplasty guidewire with the X-Sizer thromboatherectomy catheter during rescue angioplasty. Rev Esp Cardiol. 57(2): 180-3 (2004).

5. Kim TJ, Kim JK, Park BM, et al. Fatal subacute stent thrombosis induced by guidewire fracture with retained filaments in the coronary artery. Korean Circ J. 43(11): 761-5 (2013).

6. Chang T-MT, Pellegrini D, Ostrovsky A, Marrangoni AG. Surgical management of entrapped percutaneous transluminal coronary angioplasty hardware. Tex Heart Inst J. 29(4): 329-32 (2002).

7. Karabulut A, Daglar E, Cakmak M. Entrapment of hydrophilic coated coronary guidewire tips: which form of management is best? Cardiol J. 17(1): 104-8 (2010). a noteworthy complication to keep in mind. Coronary artery thrombosis is the most serious complication which can occur by leaving the guidewire inside the coronary artery. Percutaneous intervention is the best option for patients. In our case we used a goose neck loop snare. This case highlights that endovascular snare system can safely and effectively be used for extraction of fractured retained guidewires inside the coronary arteries which prevents impending surgical intervention. However if guidewire retrieval is not successful the patient can be managed conservatively or surgically.

8. Pourmoghaddas $\mathrm{M}$, Fard $\mathrm{OH}$. Retained jailed wire: a case report and literature review. ARYA Atheroscler. 7(3): 129-31 (2011).

9. Foster-Smith KW, Garratt KN, Higano ST, et al. Retrieval techniques for managing flexible intracoronary stent misplacement. Cathet Cardiovasc Diagn. 30: 63-68 (1993).

10. Patel T, Shah S, Pandya R, et al. Broken guide wire fragment: a simplified retrieval technique. Catheter Cardiovasc Interv. 51: 483-486 (2000).

11. Vrolix M, Vanhaecke J, Piessens J, et al. An unusual case of guide wire fracture during percutaneous transluminal coronary angioplasty. Cathet Cardiovasc Diagn. 15: 99-102 (1988).

12. Kume K, Yasuoka Y, Sasaki T. Successful percutaneous retrieval of a guiding catheter tip that had unexpectedly become detached using a two-wire technique. Int J Angiol. 25(5): 160-62 (2016).

13. Chiang $\mathrm{CH}$, Shih CL. Successful retrieval of an entrapped rotablator burr by using a guideliner guiding catheter and a snare. Acta Cardiol Sin. 33(1): 96-98 (2017).

14. Capuano F, Simon C, Roscitano A, et al. Percutaneous transluminal coronary angioplasty hardware entrapment: guidewire entrapment. J Cardiovasc Med. 9(11): 1140-1 (2008).

15. Proctor MS, Koch LV. Surgical removal of guide wire fragment following transluminal coronary angioplasty. Ann Thorac Surg. 45: 678-679 (1988). 